
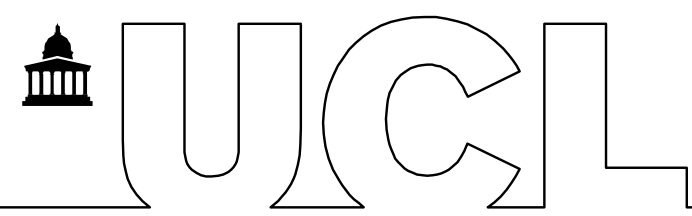

Sharma, G; Kneafsey, L; Ashley, P; Noar, J; (2015) Failure of eruption of permanent molars: a diagnostic dilemma. International Journal of Paediatric Dentistry 10.1111/ipd.12163. (In press).

Downloaded from UCL Discovery: http://discovery.ucl.ac.uk/1468940

\title{
ARTICLE
}

\section{Failure of eruption of permanent molars: a diagnostic dilemma}

\author{
Sharma, Geetanjali; Kneafsey, Louise; Ashley, Paul; Noare, Joseph \\ Eastman Dental Institute, University College London
}

\begin{abstract}
Background: Limited studies have proposed protocols for the management of failure of eruption (FOE) of permanent molars with no clear consensus or guidelines. Accurate diagnosis is challenging but key for successful management.
\end{abstract}

Aims: Confirm key diagnostic criteria that will aid diagnostic differentiation between primary failure of eruption (PFE) and mechanical failure of eruption (MFE) of permanent molars and identify successful management strategies.

Design: Retrospective descriptive study. Patients diagnosed with FOE of permanent molars (excluding impaction) between January 2003 and December 2013 were identified. Patient details, clinical and radiological features of FOE were recorded. Two examiners independently analysed the data. A diagnosis of PFE or MFE was made based on a combination of current protocols. Management strategies for each were identified and reported as satisfactory/unsatisfactory.

Results: 31 patients met the inclusion criteria. 15 were classified as PFE and 14 as MFE. 1 patient was too young to confirm diagnosis, 1 patient had delayed eruption. $26 \%$ of cases were misdiagnosed which led to unsuccessful orthodontic management.

Conclusion: We propose a protocol based on the results of this study coupled with existing protocols in the form of a simple flow diagram to aid accurate diagnosis and management of this rare and challenging clinical problem.

\section{Introduction}

Failure of eruption (FOE) of first and second permanent molars is rare, with an estimated prevalence of $0.01 \%$ in the case of the first permanent molar and $0.06 \%$ in the case of the second ${ }^{1}$. When there is failure of eruption of a permanent molar the following differential diagnoses should be considered in addition to tooth impaction: Primary failure of eruption (PFE) (Type I/ Type II) and single tooth ankylosis or mechanical failure of eruption (MFE).

PFE is an isolated condition causing a localised failure of tooth eruption with no other identifiable local or systemic involvement. It has been described based on its clinical appearance and includes the following features: it primarily affects posterior teeth; it affects all teeth posterior to the most anteriorly affected tooth; the occlusion manifests as a lateral open bite; teeth fail to respond to orthodontic forces ${ }^{2}$. Frazier-Bowers et al. ${ }^{3}$ described three different forms of PFE. One group was classified as Type I, in which all affected teeth had a similar lack of eruption potential with a progressive open bite from anterior to posterior. A 
second group was classified as Type II in which a subject had a tooth distal to the most mesial affected tooth with greater although inadequate eruption showing a more varied eruption potential among the affected teeth (Figure 1). A third group was identified in which subjects had both types of PFE coexisting in different quadrants.

The aetiology of PFE is complex. Evidence from the literature indicates that this disorder has a strong genetic component. A strong family history has been found in many studies ${ }^{4-7}$. Further, an association between PFE with dental anomalies of known genetic origin would also suggest that PFE has a substantial genetic component ${ }^{8}$. For example Mattheeuws et al. ${ }^{9}$ found the level of hypodontia to be considerably higher in the PFE population at $13 \%$ than that of the normal population. Recent studies have shown that a genetic mutation in the PTH1R gene is also associated with PFE in which the mutation is present in multiple numbers of some families who exhibit PFE ${ }^{10-12}$. In this regard, a positive family history +/confirmed mutation of PTH1R gene can be used to aid diagnosis of PFE. Hormonal disturbances or developmental syndromes such as cleidocranial dysplasia, Osteopetrosis and GAPO syndrome have also been associated with PFE in which abnormal eruptive mechanisms can delay or prevent tooth eruption ${ }^{13 .}$

Single tooth ankylosis/MFE is a rare condition with a similar presentation to PFE. A key diagnostic feature distinguishing PFE type II from MFE is that MFE often only affects a single tooth and teeth distal to that are not affected. This is difficult to diagnose in a young child, as it is impossible to determine whether all the teeth distal to the first permanent molar will suffer the same fate. Clinically, where a tooth is partially erupted the percussion test can be carried out and a tooth affected by MFE is likely to exhibit a dull metallic sound on percussion ${ }^{14}$. Adjacent teeth may tilt into the space and opposing teeth can over-erupt. Radiographically, there is appearance of relative submergence due to ankylosis and the eruptive path is not clear. A focal obliteration of the periodontal ligament space or resorption of the root surface may be seen although this may not be obvious on the radiographs as it may occur on the buccal or lingual aspects of the tooth, or, because it occurs minimally on the mesial and distal aspects ${ }^{15}$.

Management of PFE is particularly challenging. Treatment options are limited and complicated by the fact that diagnosis of the condition relies on a method of exclusion, where all other possible causative factors have been considered and eliminated rather than a positive finding. No treatment is often the best option, accepting the position of the affected teeth. Surgical extraction of the affected teeth followed by prosthetic replacement is another option however, extensive bone loss may warrant a bone graft prior to implant placement. A localized bony osteotomy and orthodontic extrusion of the entire segment has been advocated to bring the tooth/teeth into occlusion but there is limited evidence of success with this approach in the literature ${ }^{16}$. Coronal build up or onlays of the affected teeth is an option with type II PFE providing an acceptable occlusion can be achieved after vertical growth of the patient has ceased ${ }^{17}$. A removable prosthesis over the affected teeth may be more predictable in achieving an acceptable occlusion ${ }^{18}$. Exposure and bonding of teeth affected by failure of eruption attributed to PFE is not advised as treatment via active orthodontic forces has been suggested to lead to localized ankyloses ${ }^{2}$. Since an ankylosed tooth is a perfect orthodontic anchor, any effort to extrude will result in the intrusion of normal teeth. Management of multiple teeth affected by PFE is deemed more difficult and the only suggested method of bringing them into occlusion is a segmental osteotomy and a bone graft interposed between the segment and the basal alveolar bone ${ }^{2,13}$. Finally, distraction osteogenesis has been reported to correct an extreme posterior open bite ${ }^{19}$. At best, an acceptable occlusion will be achieved and at the worst the teeth will be moved into a more favourable position to aid prosthetic management. 
Making an early distinction between MFE and PFE is important in the management of patients with failure of eruption of permanent molars since they dictate different treatment modalities. MFE is often successfully treated via the extraction of the affected tooth at the appropriate age or occasionally luxation of the affected tooth and subsequent orthodontic alignment, which would be futile in cases of PFE.

Frazier-Bowers et al. ${ }^{3}$ and Rhoads et al. ${ }^{20}$ have attempted to make a distinction between PFE and MFE. The latter study suggests clinical parameters that can be utilised to diagnose PFE. These include the involvement of the first permanent molar and supracrestal presentation of the affected teeth $+/$ - involvement of the second premolar and second molar, multiple adjacent teeth, bilateral presentation, involvement of teeth in both arches, class III malocclusion and concurrent dental anomalies. They found that some features diagnostic of PFE were also common in their MFE cases hence, in their 'decision tree' they place emphasis on the identification of a mutation in the PTH1R gene as a diagnostic tool. This diagnostic test is not widely accessible but, it should not be eliminated as an option in diagnosis since the advent of genetic variation in humans is a reality in a responsible healthcare society. In comparison, Frazier- Bowers et al. ${ }^{3}$ divide their sample into one of four classifications and specify different diagnostic criteria. Specifically, cases were classified as PFE if there was a clear eruption path on a panaromic radiograph, if teeth distal to the most mesial were also affected and if any or all posterior quadrants were involved. MFE was diagnosed if there was a radiographic appearance of submergence due to ankylosis, no clearance of eruption path and when teeth distal to the most mesial affected tooth were apparently normal. This highlights the difficulty in diagnoses and often due to varying characteristics a definitive diagnosis might only be made retrospectively, once the patient is dentally mature.

To summarise, accurate diagnosis of failure of eruption is challenging with evidence that PFE is sometimes misdiagnosed as MFE ${ }^{11},{ }^{21}$. Misdiagnosis and mismanagement of either can lead to inappropriate and extended treatment times, reduced patient satisfaction, significant financial burden and risk of an inferior occlusal result. There is currently no clear consensus in the diagnosis of failure of eruption and there is limited information regarding successful management strategies in the literature. Hence, the aim of this study was to confirm key diagnostic criteria that will aid diagnostic differentiation between PFE and MFE of permanent molars and identify successful management strategies based on a 10 year retrospective review of cases diagnosed with failure of eruption at the Eastman Dental Hospital, UCLH coupled with previously published protocols.

\section{Materials and Methods}

\section{Study subjects}

This study was a retrospective analysis of patient records from the dental hospital. All patients referred to the orthodontic department of the Eastman Dental Hospital, UCLH London and diagnosed with failure of eruption of permanent molar(s) through the joint orthodontic-paediatric or orthognathic clinics between 2003 and 2013 were identified from the clinic daybooks. Subjects meeting the following inclusion criteria were included in the study: un-erupted or arrested eruption of a permanent molar tooth or teeth and no evidence of physical obstruction to eruption. Subjects exhibiting a medical history of a syndrome or in which deciduous teeth only were affected were not included.

Data Collection and analysis

The following data was extrapolated and recorded using an Excel 2007 spreadsheet:

1) Age

2) Gender

3) Ethnicity

4) Diagnosis: PFE type I or II/ MFE 
5) Unilateral/bilateral presentation

6) Single arch or both arches

7) Teeth involved - single or multiple, deciduous or permanent

8) Supracrestal or infracrestal

9) Skeletal pattern

10) Radiographical appearance of eruption pathway

11) Management strategy/treatment conducted

12) Outcome

13) Presence of other associating dental anomalies e.g. supernumerary teeth, ectopia, agenesis, microdontia/macrodontia cementomas

Patient records including clinical entries, correspondences, photographs and radiographs (OPG +/- Lateral Cephalogram) when available were examined independently by two examiners (GS, LK) and a diagnosis of MFE or PFE was made based on the diagnostic criteria that are enlisted in Table 1. If there was a disagreement in diagnosis between both examiners the subject in question was recalled to confirm a diagnosis with a third examiner (JN). The diagnostic criteria enlisted in Table 1 stems from a literature review discussed in the introduction ${ }^{3,20}$. A literature search was performed using MEDLINE and PUBMED. The authors aimed to identify all studies in which the diagnosis and or management of failure of eruption of permanent molars were discussed. Abstracts from the electronic searches were used to create a list of potentially relevant studies. Thereafter, the full text of all potentially relevant papers were obtained and reviewed for inclusion. Articles in which subjects had failure of eruption of any other teeth except permanent molars or failure of eruption of permanent molars attributed to impaction were excluded.

\section{Results}

Study Subject characteristics

Of those identified from the clinics 31 patients satisfied the inclusion criteria. Of these 15 were diagnosed with PFE and 14 with MFE. One patient was deemed to have simple delayed eruption. One patient was too young (at 8 years old) to make a definitive diagnosis and was to be reviewed in 1 year to assess development of the more posterior teeth.

The demographic details of the patients are summarised in Table 2. The age range at initial presentation was wide for both MFE (aged $9-19$ years) and PFE (aged $6-55$ years). Males were more commonly affected than females. A number of different ethnicities were affected. No family history was recorded for any patient in either group.

Table 3 summarises the clinical features that were present in the MFE and PFE cohorts coupled with their prevalence. Predominant features amongst the MFE and PFE patients are highlighted in bold. All cases of MFE were unilateral, confined to a single arch and affected a single tooth (100\%). The first permanent molar was affected in $71 \%$ of cases and the second permanent molar affected in $29 \%$ cases with MFE. The deciduous dentition was not affected. In $57 \%$ of cases the affected tooth was unerupted. Cases with MFE were more likely to have a Skeletal Class I $(n=9)$.

Radiographically, 4 cases with MFE had bone superior to the crown of the tooth and the eruption pathway was not clear. Congenitally absent upper second premolars, short roots of upper first premolars, short sclerosed root form or delayed/poor root form of the affected molar tooth, cementoma and impacted teeth were additional dental anomalies found amongst subjects diagnosed with MFE.

The management of cases with MFE was split between accepting the position of the affected tooth $(n=3)$ or surgical extraction of the affected tooth (with or without extraction of the opposing tooth $(n=9)$. One patient had extraction of the affected tooth in addition to other 
orthodontic extractions and comprehensive orthodontic correction of the malocclusion. One patient failed to attend their follow up appointments.

PFE had a more varied presentation with $47 \%$ of cases affected bilaterally and $60 \%$ where both the maxilla and the mandible were affected. PFE affected multiple teeth in $100 \%$ of cases. In $100 \%$ of cases the first permanent molars were affected, whilst the second molars were affected in $93 \%$ of cases. The premolars were affected in $64 \%$ of cases and the deciduous dentition in $21 \%$ of cases. Teeth affected by PFE were unerupted or partially erupted. A Skeletal III base $(n=7)$ was most prevalent in cases with PFE followed by a Skeletal II base $(n=5)$.

Radiographically $100 \%$ of cases with PFE had a clear eruption pathway superior to the unerupted tooth. Hypercementosis, significantly curved root formation, presence of a supernumerary tooth, delayed root development/short roots were features noted in subjects with PFE.

Management of the cases with PFE was more complex. 3 patients chose to accept the position of their teeth and sought no further treatment. One patient had overdentures provided to improve their posterior occlusion. One case had orthodontic alignment of the upper and lower labial segments only to improve a Class III incisor relationship, which had the added benefit of allowing the mandible to autorotate and reduce the posterior lateral open bites; subsequently, the teeth affected by PFE were more easily restorable. 4 cases had unsuccessful orthodontic alignment following exposure and bonding of the affected teeth. 5 cases had the affected teeth surgically removed and then subsequently the more distal teeth failed to erupt also. 1 case failed to attend follow up.

\section{Discussion}

There is a lack of distinctive diagnostic criteria available in the literature that can be used to aid accurate diagnosis between PFE and MFE, making it challenging for clinicians to make an accurate diagnosis and appropriate treatment plans thereafter.

By retrospectively evaluating all our patients who have been diagnosed with failure of eruption of permanent molars over the last 10 years coupled with a literature review we hoped to formulate definitive diagnostic criteria and identify successful management strategies for both PFE and MFE, as there is currently no gold standard.

Most of the clinical features of PFE observed in our sample have also been reported in other studies and include involvement of $1^{\text {st }}$ permanent molar, multiple adjacent teeth affected, involvement of second premolar and molar, supracrestal presentation, bilateral presentation (in most cases), involvement of teeth in both arches, frequent Class III malocclusion and high prevalence of concurrent dental anomalies. Similarly, there are features of MFE found in this study that are in keeping with the existing literature. These include single tooth involvement, unilateral presentation, involvement of teeth in a single arch, supracrestal and infracrestal presentation and presence of concurrent dental anomalies.

In our study, the first permanent molar was always affected in subjects with PFE and in only $75 \%$ of MFE cases. This observation of first permanent molar involvement is also reported in the study by Rhoads et al. ${ }^{20}$ however, in their cohort of subjects first permanent molar involvement was also reported in $100 \%$ of their MFE subjects although this was based on a smaller sample size $(n=6)$.

Supracrestal and infracrestal presentation was present for both MFE and PFE subjects but infracrestal presentation of MFE was more common at $66 \%$. Rhoads et al. ${ }^{20}$ only reported infracrestal presentation of MFE in one subject. 
In keeping with Rhoads et al. $^{20}$ the affected tooth was confined to a single arch with unilateral presentation in every case for MFE which is in stark contrast to PFE cases in which the majority $(60 \%)$ of subjects had features in both arches with bilateral presentation.

Other features that were observed comparatively more in PFE subjects was that of Class III pattern at $46 \%$. This has also been observed in other studies ${ }^{2,20,3}$. Since there appears to be an association between PFE and Class III malocclusion it has been speculated that there may be a generalized disturbance in bone metabolism and turnover that affects the growth and development of the maxilla as well as the dentition. A strong family history of Class III malocclusions in cases with PFE has been demonstrated in previous research hence there may be an overlapping genetic component to dental and skeletal disturbances ${ }^{22}$. Unfortunately, in this cohort of patients family history of eruption disturbances could not be confirmed from the available data.

Concurrent dental anomalies were observed in both PFE and MFE subjects with an increased prevalence amongst the PFE subjects which, is in keeping with the literature ${ }^{20,3}$, suggesting that both eruption disorders are under some form of genetic control.

The management of cases with MFE was divided between no treatment, surgical removal of the affected tooth +/- opposing tooth and adjunctive orthodontic treatment if necessary. The treatment of these cases can be considered successful based on spontaneous eruption of all the teeth distal to the affected tooth into an acceptable position or successful orthodontic alignment.

The management of cases with PFE included unsuccessful orthodontic alignment following exposure and bonding $(n=4)$. This is in keeping with former studies in the literature which have discussed that active intervention in these cases is unsuccessful placing an unnecessary treatment burden and its associated risks upon the patient ${ }^{3,23,20}$. Similarly, early surgical removal of the affected $1^{\text {st }}$ permanent molar $(n=5)$ did not yield any great benefit for these patients since the teeth distal subsequently also failed to erupt. Hence, premature treatment did not benefit this cohort of patients. This is exactly why more guidance is required when faced with this diagnostic dilemma. The remainder of the subjects affected by PFE $(n=6)$ chose to either accept the occlusion or were managed via restorative treatment with or without adjunctive orthodontic treatment, which led to a satisfactory outcome, based on successful occlusal contacts with opposing teeth and patients ability to function.

We acknowledge that there are limitations to this study. Firstly, this is a retrospective study and although this type of study design has the advantage that it is based on existing data a prospective multi-centre study would be optimal. However, difficulties with recruitment would be encountered due to the rarity of the condition. This is reflected by the fact that existing studies in the literature are also retrospective.

The sample size is also a limitation, but understandable as failure of eruption of permanent molars (excluding impaction) is rare. Hence, a retrospective study over a 10 year period was conducted in order to maximise the sample size as much as possible. Future involvement of other hospitals would prove beneficial in this regard.

The diagnostic criteria that were used to diagnose PFE or MFE is listed in table 1 and stems from reported clinical features of these eruption disorders and diagnostic criteria used in previous studies ${ }^{3,10}$. We felt that since there is a lack of clear definitive diagnostic criteria in the literature this would be the most robust and descriptive measure for diagnosis. 
Clinical notes, including photographs and radiographs when available, were used to diagnose PFE or MFE. This was dependant on detailed and accurate record keeping. Clinical examination of all the patients might be deemed more desirable.

In summary, whilst the results of this study and former studies have highlighted that both PFE and MFE affect the first permanent molar and can be infracrestal or supracrestal in their presentation there are distinctions between the clinical features of PFE and MFE which are highlighted in figure 2 which, can be utilised by clinicians to aid diagnosis of PFE and MFE.

\section{Why this paper is important to paediatric dentists?}

- MFE and PFE are rare conditions and similar in their presentation, especially in the mixed dentition stages. Differentiating between the two is paramount in making the correct diagnosis and managing the case successfully.

- Data from a 10-year retrospective audit and literature review has been used to create a user-friendly flow diagram to help with the diagnostic dilemma of failure of eruption of permanent molar teeth. Appropriate treatment options are also offered, which should ultimately lead to more efficient and effective management.

\section{Conflict of interest}

The authors declare no conflict of interest.

\section{References}

1. Grover PS, Lorton L. The incidence of unerupted permanent teeth and related clinical cases. Oral Surg Oral Med Oral Pathol 1985; 59: 420-25Ireland AJ. Familial posterior open bite: A primary failure of eruption. Br J Orthod 1991; 18: 233-237

2. Proffit WR, Vig K. Primary failure of euption. A possible cause of posterior open bite. Am J Orthod 1981; 80: 173-190

3. Frazier-Bowers SA, Koehler KE, Ackerman JL, Proffit WR. Primary failure of eruption: further characterization of a rare eruption disorder. Am J Orthod Dentofacial Orthop $2007 ; 131: 578$

4. Reid D 1954. Incomplete eruption of the first permanent molar in two generations of the same family. British Dental Journal 96: 292-294

5. Brady J. Familial primary failure of eruption of permanent molar teeth. $\mathrm{Br} \mathrm{J}$ Orthod 1990; 17: 109-113

6. Ireland AJ. Familial posterior open bite: a primary failure of eruption. $\mathrm{Br} J$ Orthod 1991; 18: 233-7

7. Dibiase AT, Leggat TG. Primary failure of eruption in the permanent dentition of siblings. Int J Paediatr Dent 2000; 10: 153-157.

8. Baccetti T. Tooth anomalies associated with failure of eruption of first and second permanent molars. Am J Orthdod Dentofac Orthop 2000; 118: 608-610

9. Mattheeuws N, Dermaut L, Martens G. Has hypodontia increased in Caucasians during the $20^{\text {th }}$ Century? A meta-analysis. Eur J Orthod 2004; 26: 99-103

10. Decker E, Stellzig-Eisenhaurer A, Fiebig BS, Rau C, Kress W, Saar K et al. PTHR1 loss-of-function mutations in familial, nonsyndromic primary failure of tooth eruption. $A m J$ Hum Genet 2008; 83:781-6

11. Frazier-Bowers SA, Simmons D, Wright JT, Proffit WR, Ackerman JL. Primary failure of eruption and PTH1R; the importance of a genetic diagnosis for orthodontic treatment planning. Am J Orthod Dentofacial Orthop 2010; 137: 160: 1-7

12. Yamaguchi T, Hosomichi K, Narita A, Shirota T, Tomoyasu Y, Maki K et al. Exome resequencing combined with linkage analysis identifies novel PTH1R variants in primary failure of tooth eruption in Japanese. J Bone Miner Res 2011; 26: 1655-61

13. Piattelli A, Eleuterio A. Primary failure of eruption. Acta Stomatology 1991; 88: 127130 
14. Proffit WR, Katherine WL. Primary failure of eruption: A possible cause of posterior open-bite. Am J Orthod Dentofacial Orthop 1981; 80: 173-190

15. Oliver RG, Hunter B. Submerged permanent molars: four case reports. Br Dent $J$ 1986; 160: 128-130

16. Takafumi S, Masako M, Yuji O, Miyuki S, Tsuyoshi T. Segmental alveolar distraction for the correction of unilateral open-bite caused by multiple ankylosed teeth: A case report. $J$ Orthod 2006; 33: 153-159

17. Yatani H, Watanabe EK, Kaneshima T, Yamashita A, Suzuki K. Etched-porcelain resin-bonded onlay technique for posterior teeth. J Esthet Dent 1998; 10: 325-32

18. Seigel SC, O' Connell A. Oral rehabilitation of a child with primary failure of tooth eruption. J Prosthodont 1999; 8: 201-207

19. Kater WM, Kawa D, Schafer D, Toll D. Treatment of posterior open bite using distraction osteogenesis. J Clin Orthod 2004; 38: 501-504

20. Rhoads SG, Hendricks HM, Frazier-Bowers SA. Establishing the diagnostic criteria for eruption disorders based on genetic and clinical data. Am J Orthod Dentofacial Orthop 2013; 144: 194-202

21. Frazier-Bowers SA. Primary failure of eruption: clinical implication of a genetic disorder. Effective and efficient tooth movement: evidence based orthodontics. Craniofacial Growth Series. Am Arbor 2011; Monograph 48

22. Proffit WR, Fields HW Jr, Moray LJ. Prevalence of malocclusion and orthodontic treatment need in the United States: estimates from the NHANES III survey. Int $J$ Adult Orthod Orthognath Surg 1998: 13: 97-106

23. Stellzig-Eisenhauer A, Decker E, Meyer PM, Rau C, Fiebig BS, Kress W et al., Primary Failure of Eruption (PFE) - Clinical and Molecular Genetics Analysis. J Orofac Orthop 2010; 71:6-16

24. Raghoebar GM, Boering G, Vissink A, Stegenga B. Eruption disturbances of permanent molars: a review. J Oral Pathol Med 1991a; 20:159-166

Figure 1 (a) Clinical presentation of a subject with failure of eruption of the mandibular $1^{\text {st }}$ and 2nd molars giving rise to a posterior open bite, indicative of PFE Type II

Figure 1 (b) Radiographical presentation of a subject with failure of eruption of the molar teeth in the lower right quadrant (PFE type II)

Figure 2 Flow-diagram to aid diagnosis and management of failure of eruption of the permanent molar tooth/teeth 
Table 1

Diagnostic criteria developed from the literature review used to distinguish PFE from MFE

\begin{tabular}{|c|c|c|c|c|}
\hline \multirow{9}{*}{$\begin{array}{l}\text { Diagnostic } \\
\text { criteria } \\
\text { Clinical }\end{array}$} & & PFE I & PFE II & MFE \\
\hline & $\begin{array}{l}\text { Permanent } \\
\text { molar affected }\end{array}$ & $\begin{array}{l}1^{\text {st }} \text { molar } \\
\text { always }\end{array}$ & $1^{\text {st }}$ molar always & $1^{\text {st }}$ or $2^{\text {nd }}$ molar \\
\hline & $\begin{array}{l}\text { Teeth distal to } \\
\text { tooth affected }\end{array}$ & Yes & Yes & No \\
\hline & $\begin{array}{l}\text { Deciduous } \\
\text { dentition } \\
\text { invoved }\end{array}$ & Possible & Possible & No \\
\hline & Multiple teeth & Possible & Possible & No \\
\hline & $\begin{array}{l}\text { Position of } \\
\text { tooth }\end{array}$ & Unerupted & $\begin{array}{l}\text { Erupted (not } \\
\text { fully) or } \\
\text { unerupted }\end{array}$ & $\begin{array}{l}\text { Erupted (not fully) or } \\
\text { unerupted }\end{array}$ \\
\hline & $\begin{array}{l}\text { Quadrants } \\
\text { involved }\end{array}$ & $\begin{array}{l}\text { Single or } \\
\text { multiple }\end{array}$ & $\begin{array}{l}\text { Single or } \\
\text { multiple }\end{array}$ & Single \\
\hline & $\begin{array}{l}\text { Posterior or lat } \\
\text { open bite }\end{array}$ & Yes & Yes & No \\
\hline & $\begin{array}{l}\text { Percussion } \\
\text { test }\end{array}$ & $\mathrm{N} / \mathrm{A}$ & $\begin{array}{l}\text { Can be negative } \\
\text { for ankylosis }\end{array}$ & Positive for ankylosis \\
\hline Radiographic & $\begin{array}{l}\text { Eruption } \\
\text { pathway }\end{array}$ & Clear & Clear & Possible bone overlying \\
\hline Other & $\begin{array}{l}\text { Response to } \\
\text { Treatment }\end{array}$ & $\begin{array}{l}\text { Unsuccessful } \\
\text { orthodontic } \\
\text { extrusion }\end{array}$ & $\begin{array}{l}\text { Unsuccessful } \\
\text { orthodontic } \\
\text { extrusion }\end{array}$ & $\begin{array}{l}\text { Successful luxation and } \\
\text { subsequent extrusion of the } \\
\text { tooth into the line of the arch } \\
\text { or extraction of affected } \\
\text { tooth and eruption of all } \\
\text { teeth distal }\end{array}$ \\
\hline
\end{tabular}

Table 2

Demographic features of the subjects

\begin{tabular}{|lll|}
\hline & MFE $\mathrm{n}=14$ & PFE $\mathrm{n}=15$ \\
Age range at presentation & $9-19$ years & $6-55$ years \\
Gender & 1.5 Male: 1 Female & 1.3 Male: 1 Female \\
Ethnicity & Caucasian & Caucasian $\mathrm{n}=9$ \\
& African & African $\mathrm{n}=1$ \\
& Turkish & Turkish $\mathrm{n}=1$ \\
& Indian & Carribean $\mathrm{n}=1$ \\
& & Indian $\mathrm{n}=2$ \\
Family History & None recorded & Pakistani $\mathrm{n}=1$ \\
\hline
\end{tabular}


Table 3

Breakdown of the clinical diagnostic features present in the total sample and in MFE and PFE populations.

\begin{tabular}{|c|c|c|c|c|c|}
\hline 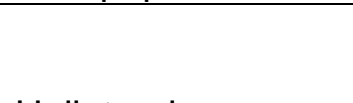 & $\mathrm{N}=29$ & MFE $n=14$ & $\begin{array}{l}\text { Prevalence } \\
\text { in MFE (\%) }\end{array}$ & PFE $n=15$ & $\begin{array}{l}\text { Prevalence } \\
\text { in PFE (\%) }\end{array}$ \\
\hline Unilateral & 22 & 14 & 100 & 8 & 53 \\
\hline Bilateral & 7 & 0 & 0 & 7 & 47 \\
\hline Single arch & 20 & 14 & 100 & 6 & 40 \\
\hline Both arches & 9 & 0 & 0 & 9 & 60 \\
\hline $\begin{array}{l}\text { Single tooth } \\
\text { affected }\end{array}$ & 15 & 14 & 100 & 1 & 6 \\
\hline $\begin{array}{l}\text { Multiple teeth } \\
\text { affected }\end{array}$ & 15 & 0 & 0 & 15 & 100 \\
\hline $\begin{array}{l}\text { First Permanent } \\
\text { Molar affected }\end{array}$ & 25 & 10 & 71 & 15 & 100 \\
\hline Premolar affected & 9 & 0 & 0 & 9 & 64 \\
\hline $\begin{array}{l}\text { Second Permanent } \\
\text { Molar affected }\end{array}$ & 18 & 4 & 29 & 14 & 93 \\
\hline $\begin{array}{l}\text { Deciduous teeth } \\
\text { affected }\end{array}$ & 3 & 0 & 0 & 3 & 21 \\
\hline Unerupted & 14 & 8 & 57 & 6 & 40 \\
\hline Erupted & 17 & 6 & 43 & 11 & 73 \\
\hline Skeletal Class & & $\begin{array}{l}I=9 \\
I I=4 \\
I I I=1\end{array}$ & & $\begin{array}{l}I=2 \\
I I=5 \\
I I I=7 \\
\text { Unknown } \\
=1\end{array}$ & \\
\hline
\end{tabular}

